\title{
INDEX OF VOLUME XXIX
}

Adams, E. P. See Reviews, under Institut, Pringsheim, Rougier.

Bateman, H. An Electromagnetic Theory of Light-Darts, 385.

BeLl, E. T. Square-Partition Congruences, 349.

- Analogies between the $u_{n}, v_{n}$ of Lucas and Elliptic Functions, 401.

Bernstein, B. A. Reports of Meetings of the San Francisco Section: October Meeting, 10; April Meeting, 193. A Correction, 33.

BLIss, G. A. The Reduction of Singularities of Plane Curves by Birational Transformation, 161.

See Reviews, under Blaschke.

Bray, H. E. Proof of a Formula for an Area, 264.

Brown, E. W. See Reviews, under Annuaire.

CAJorI, F. The Name "Divergent" Series, 55.

- See Reviews, under Andoyer, Iamblichus, Monge.

Carmichael, R. D. See Reviews, under Lie.

Carslaw, H. S. The Differentiation of a Function of a Function, 344.

Coble, A. B. See Reviews, under Malet.

Coolidge, J. L. See Reviews, under Beck.

Cowley, E. B. See Reviews, under Liebmann, MacLeod, Müller.

CraIG, C. F. On the Riemann Zeta Function, 337.

Crathorne, A. R. See Reviews, under Charlier.

Curtiss, D. R. Relations between Kindred Riemannian $P$ and $Q$ Functions, 154.

Danield, P. J. See Reviews, under Grammel, Kennelly, Milankovitch, Page.

Davis, H. T. See Reviews, under Cartan.

Dickson, L. E. Integral Solutions of $x^{2}-m y^{2}=z w, 464$.

Douglas, J. Determination of all Systems of $\infty 4$ Curves in Space in which the Sum of the Angles of every Triangle is Two Right Angles, 356.

Dowling, L. W. See Reviews, under Amodeo, Fricke.

Dresden, A. Reports of Meetings of the Chicago Section: December Meeting, 117; April Meeting, 197.

Eмch, A. See Reviews, under Groll, Ling.

Etthinger, H. J. An Elementary Proof of a Fundamental Lemma concerning the Limit of a Sum, 219.

Fischer, C. A. See Reviews, under Lévy.

Forsyth, C. H. See Reviews, under Andoyer, Glover, Soper.

Frankin, P. A Qualitative Definition of the Trigonometric and Hyperbolic Functions, 56.

GABA, M. G. A Set of Axioms for Line Geometry, 128.

Glover, J. W. See Reviews, under Richard.

Graustein, W. C. Note on a certain Type of Ruled Surface, 341.

- See Reviews, under de Tannenberg.

HILDEBRANDT, T. H. On Uniform Limitedness of Sets of Functional Operations, 309. 
- See Reviews, under Knopp.

Hruton, H. A Generalization of a Property of an Acnodal Cubic Curve, $303,417$.

Hollcroft, T. R. Singularities of Curves of Given Order, 407.

INGOLD, L. See Reviews, under Bieberbach. .

$\mathrm{J}_{\mathrm{ACKSON}}, \mathrm{D}$. Note on Quartiles and Allied Measures, 17.

- Note on the Convergence of Weighted Trigonometric Series, 259.

Karpinski, L. C. See Reviews, under Gunther, Wieleitner.

KellogG, O. D. See Reviews, under Hurwitz.

Kempner, A. J. See Reviews, under Bachmann, Perron.

Lamson, K. W. See Reviews, under Eddington.

Lane, E. P. See Reviews, under Cartan.

Lefschetz, S. Report on Curves Traced on Algebraic Surfaces, 242.

Lehmer, D. N. See Revirws, under Cunningham.

LIPKA, J. On the Relative Curvature of Two Curves in $V_{n}, 345$.

- See Reviews, under Ensslin.

Lytle, E. B. See Reviews, under Branford.

McClenon, R. B. See Reviews, under Archimedes, Fermat, Tropfke, Tweedie.

MACDUfFee, C. C. On Transformable Systems and Covariants of Algebraic Forms, 26.

MacMillan, W. D. See Reviews, under Goursat, Schlesinger.

Miller, G. A. Groups in which the Number of Operators in a Set of Conjugates is Equal to the Order of the Commutator Subgroup, 64.

- Same Left Co-Set and Right Co-Set Multipliers for any given Finite Group, 394.

- See Reviews, under Speiser.

Moore, C. L. E. See Reviews, under Planck.

Moore, C. N. See Reviews, under Carslaw.

Moore, R. L. An Uncountable, Closed, and Non-Dense Point Set each of whose Complementary Intervals Abuts on Another One at each of its Ends, 49.

- Report on Continuous Curves from the Viewpoint of Analysis Situs, 289.

Mordeld, I. J. An Introductory Account of the Arithmetical Theory of Algebraic Numbers and its Recent Developments, 445.

Murray, F. H. Periodic Solutions in the Problem of Three Bodies, 15.

Phillips, H. B. See Reviews, under Gray, Möller, Shaw.

Poritsky, H. On Curves Kinematically Related to a given Curve, 71 .

Porter, M. B. The Second Mean Value Theorem for Summable Functions, 399.

Reynolds, C. N. See Reviews, under Fournier.

Richardson, R. G. D. Reports of Meetings of the American Mathematical Society: October Meeting, 1; Twenty-Ninth Annual Meeting, 97; February Meeting, 145; April Meeting in New York, 204; Thirtieth Summer Meeting, 433.

- The Frank Nelson Cole Prize in Algebra, 14.

- Incorporation of the American Mathematical Society, 241. 
The Josiah Willard Gibbs Lectureship, 385.

On the Reality of the Zeros of a Lambda Determinant, 467.

Rretz, H. L. See Reviews, under Czuber, Glover, Insolera.

Rowe, J. E. See Reviews, under Ordnance Department, Vahlen.

SHaw, J. B. See Reviews, under Boussinesq, Juvet, Valentiner, Weatherburn.

Silverman, L. L. See Reviews, under Bolzano.

Sisam, C. H. See Revrews, under Pascal.

Smith, D. E. See Reviews, under Archimedes, Heath.

SNyder, V. See Reviews, under Klein, Schmid.

Stouffer, E. B. Report of the Fifteenth Regular Meeting of the Southwestern Section, 125.

Swirt, E. See Reviews, under Runge.

Whittemore, J. K. Ruled Surfaces with Director Planes, 21.

Total Geodesic Curvature and Geodesic Torsion, 51.

Wilson, E. B. See Reviews, under Keynes, Vance.

Young, J. W. See Reviews, under Boutroux, Granville, Keyser, Kowalewski, Michel, Neville, Riemann, Weber.

Young, J. W. A. See Reviews, under Lietzmann.

\section{Reviews}

Amodeo, F. Lezione di Geometria Proiettiva (terza edizione), L. W. Dowling, 85.

Andoyer, H. L'Oeuvre Scientifique de Laplace, F. CAJORI, 232.

- Tables Logarithmiques à Treize Décimales, C. H. Forsyth, 373.

Annuaire du Bureau des Longitudes pour l'An 1922, E. W. Brown, 89.

Archimedes. Les Oeuvres Complètes, traduites du Grec en Français par Paul Ver Eecke, D. E. Sмiтн, 274.

- Kugel und Zylinder, übersetzt und mit Anmerkungen versehen, von Arthur Czwalina-Allenstein, D. E. SмIтн, 274.

- Ueber Spiralen, übersetzt von Arthur Czwalina-Allenstein, R. B. McClenon, 473.

Bachmann, P. Grundlehren der Neueren Zahlentheorie, A. J. Kempner, 36.

Beck, H. Koordinaten Geometrie, erster Band, J. I. CoolIDGE, 316.

Bierberbach, L. Differential- und Integralrechnung, erster Band, L. INGOLD, 421.

Blaschke, W. Vorlesungen über Differentialgeometrie und Geometrische Grundlagen von Einstein's Relativitätstheorie, erster Band, G. A. BuIss, 322.

Bolzano, B. Paradoxien des Unendlichen, L. L. Silvterman, 370.

Boussinesq, J. Cours de Physique Mathématique de la Faculté des Sciences, Compléments au Tome III, J. B. Shaw, 329.

Boutroux, P. L'Idéal Scientifique des Mathématiciens, J. W. Young, 470.

Branford, B. A Study of Mathematical Education (new edition), E. B. LYTLE, 90.

Carslaw, H. S. Introduction to the Mathematical Theory of the Conduction of Heat in Solids (second edition), C. N. Moors, 326. 
Cartan, E. Leçons sur les Invariants Intégraux, E. P. LANe and H. T. Davis, 140.

Charlier, C. V. I. Vorlesungen über die Grundzüge der Mathematischen Statistik (zweite Auflage), A. R. Crathorne, 418.

Courant, R. See Hurwitz, A.

Cunningham, A. Fundamental Congruence Solutions, D. N. LEHMER, 374.

- Haupt-Exponents, Residue-Indices, Primitive Roots, and Standard Congruences, D. N. LaHMER, 374.

Czuber, E. Wahrscheinlichkeitsrechnung und ihre Anwendung auf Fehlerausgleichung, und Lebensversicherung (dritte Auflage), zweiter Band, H. L. Rietz, 39.

- Die Statistischen Forschungsmethoden, H. L. RIETz, 39.

Czwalina-Allenstein, A. See Archimedes.

Eddington, A. S. Espace, Temps, et Gravitation, K. W. Lamson, 87.

Engel, F. See Lie, S.

Ensslin, M. Elastizitätslehre für Ingenieure (zweite Auflage), erster Band, J. LIPKA, 281.

Epstein, P. See Weber, H.

de Falco, V. See Iamblichus.

de Fermat, P. Einführung in die Ebenen und Körperlichen Oerter, R. B. McClenon, 474 .

Fournier, G. La Relativité Vraie et la Gravitation Universelle, C. N. Reynolds, 479.

Fricke, R. Die Elliptischen Funktionen und ihre Anwendungen, zweiter Teil, L. W. Dowling, 234.

Glover, J. W. United States Life Tables, H. L. RIETz, 86.

Tables of Applied Mathematics in Finance, Insurance, Statistics, C. H. Forsyth, 376.

Goursat, E. Leçons sur l'Intégration des Equations aux Dérivées Partielles du Premier Ordre (seconde édition), W. D. MacMillan, 280.

Graf, O. See Groll, M.

Grammel, R. Der Kreisel, P. J. Danield, 40.

Granville, W. A. The Fourth Dimension and the Bible, J. W. Young, 185.

Gray, A., Mathews, G. B., and MacRobert, T. M. Bessel Functions, H. B. PhILLIPS, 423.

Groll, M. Kartenkunde, neu bearbeitet von Dr. Otto Graf, erster Teil, A. ЕмСH, 89.

Gunther, R. T. Early Science in Oxford, part II, L. C. KARPINSKI, 475.

Heath, T. A History of Greek Mathematics, volumes I-II, D. E. SмIтH, 79.

Heegaard, P. See Lie, S.

Hurwitz, A. Vorlesungen über Allgemeine Funktionentheorie und Elliptische Funktionen, herausgegeben und ergänzt durch einen Abschnitt über Geometrische Funktionentheorie von R. Courant, O. D. KELLOGG, 415.

Iamblichus. Theologoumena Arithmeticæ, edidit Victorius de Falco, F. CAJORI, 377.

Insolera, F. Lezioni di Statistica Metodologica, H. L. RIEtz, 90. 
Institut International de Physique Solvay, Atomes et Electrons, E. P. ADAMs, 422.

Juvet, G. Introduction au Calcul Tensoriel et au Calcul Différentiel Absolu, J. B. SHaw, 88.

Kennelly, A. E. Les Applications Elémentaires des Fonctions Hyperboliques à la Science de l'Ingénieur Electricien, P. J. DANIELL, 328.

Keynes, J. M. A Treatise on Probability, E. B. WiLson, 319.

Keyser, C. J. Mathematical Philosophy, J. W. YounG, 271.

Klein, F. Gesammelte Mathematische Abhandlungen, zweiter Band, V. SNYDER, 224.

Knopp, K. Theorie und Anwendung der Unendlichen Reihen, T. H. HILDEBRANDT, 474.

Kowalewski, G. Mathematica Delectans, erstes Heft, J. W. Young, 40.

Kruppa, E. See Müller, E.

Lévy, P. Leçons d'Analyse Fonctionnelle, C. A. Fischer, 229.

Lie, S. Gesammelte Abhandlungen, herausgegeben von Friedrich Engel und Poul Heegaard, dritte Band, erste Abteilung, R. D. Carmichaed, 367.

Liebmann, H. Nichteuklidische Geometrie (dritte Auflage), E. B. CowLEY, 424 .

Iietzmann, W. Methodik des Mathematischen Unterrichts (zweite Auflage), zweiter Teil, J. W. A. Young, 479.

Ling, G. H., Wentworth, G., and Smith, D. E. Elements of Projective Geometry, A. ЕмсH, 233.

MacLeod, A. Introduction à la Géométrie Non-Euclidienne, E. B. CowLEY, 424.

MacRobert, T. M. See Gray, A.

Malet, H. Etude Géométrique des Transformations Birationnelles et des Courbes Planes, A. B. CoBle, 38.

Mathews, G. B. See Gray, A.

Michel, F., et Potron, M. La Composition des Mathématiques dans l'Examen d'Admission à l'Ecole Polytechnique de 1901 à 1921, J. W. Young, 232.

Milankovitch, M. Théorie Mathématique des Phénomènes Produits par la Radiation Solaire, P. J. Danield, 419.

Möller, M. Kraftarten und Bewegungsformen, H. B. PhILLIPs, 421.

Monge, G. Géométrie Descriptive, F. CAJORI, 420.

Müller, E. Lehrbuch der Darstellenden Geometrie für Technische Hochschulen (dritte Auflage), zweiter Band, E. B. Cowlexy, 478.

- Vorlesungen über Darstellende Geometrie, erster Band, bearbeitet von Dr. Erwin Kruppa, E. B. CowLey, 478.

Neville, E. H. The Fourth Dimension, J. W. Young, 38.

Ordnance Department. A Course in Exterior Ballistics, J. E. Rowe, 277.

Page, L. An Introduction to Electrodynamics, P. J. DANIELL, 39.

Pascal, E. Repertorium der Höheren Mathematik (zweite Auflage), zweiter Band, zweite Hälfte, herausgegeben von H. E. Timerding, C. H. Sisam, 373.

Perron, O. Irrationalzahlen, A. J. Kempner, 34.

Petit, E. See Richard, P. J. 
Planck, M. Einführung in die Mechanik Deformierbarer Körper (zweite Auflage), C. L. E. Moone, 478.

Potron, M. See Michel, F.

Pringsheim, P. Fluoreszenz und Phosphoreszenz im Lichte der neueren Atomtheorie, E. P. ADAMs, 234.

Richard, P. J., et Petit, E. Théorie Mathématique des Assurances. (seconde édition), J. W. Glover, 139.

Riemann, B. Utber die Hypothesen welche der Geometrie zu Grunde liegen. J. W. Young, 480.

Rougier, L. La Matière et l'Energie selon la Théorie de la Relativité et la Théorie des Quanta (nouvelle édition), E. P. ADAMs, 282.

Runge, C. Praxis der Gleichungen (zweite Auflage), E. SwIFT, 327.

Schlesinger, L. Einführung in die Theorie der Gewöhnlichen Differentialgleichungen auf Funktionentheoretischer Grundlage (dritte Auflage), W. D. MAcMillan, 282.

Schmid, T. Darstellende Geometrie (dritte Auflage), erster Band, V. SNYDER, 280.

Shaw, J. B. Vector Calculus H. B. Phillips, 375.

Smith, D. E. See Ling, C. H.

Soper, H. E. Frequency Arrays, C. H. Forsyth, 422.

Speiser, A. Die Theorie der Gruppen von endlicher Ordnung, G. A. MILLER, 372.

de Tannenberg, W. Conférencess sur les Transformations en Géométrie Plane, W. C. Graustern, 87.

Timerding, H. E. See Pascal, E.

Tropfke, J. Geschichte der Elementarmathematik (zweite Auflage), erster, zweiter, dritter, und vierter Band, R. B. McClenon, 476.

Tweedie, C. James Stirling, A Sketch of his Life and Works, R. B. McClenon, 184.

Vahlen, T. Ballistik, J. E. Rowe, 186.

Valentiner, S. Vektoranalysis (dritte Auflage), J. B. SHAw, 377.

Vance, R. Business and Investment Forecasting, E. B. WILson, 281.

Ver Eecke, P. See Archimedes.

Weatherburn, C. E. Elementary Vector Analysis, J. B. SHAw, 233.

Weber, H., and Wellstein, J. Encyklopädie der Elementarmathematik (vierte Auflage), erster Band, neubearbeitet von P. Epstein, J. W. Young, 480.

Wellstein, J. See Weber, H.

Wentworth, G. See Ling, G. H.

Weyl, H. See Riemann, B.

Wieleitner, H. Geschichte der Mathematik, erster Band, L. C. KarPINSKI, 424.

Errata, 33, 417.

Index of Volume XXIX, 495.

New Publications, 43, 93, 143, 190, 238, 286, 334, 381, 429, 483.

Notes, $41,91,141,188,235,283,331,378,425,481$.

Papers Read before the Society and Subsequently Published, ThirtySecond Annual List of, 485. 


\section{Notes AND Other Items}

Academies, Associations, Congresses, and Societies:

American Mathematical Society: Annual Meeting, 99; Bôcher Prize, 188; Chicago Section, December Meeting, 197, 425; Cole Prize, 2, 14, 205; Election of Officers, 100; Endowment Fund, 100, 146, 188, 205, 283, 333, 435; Gibbs Lectureship, 385, 435; Incorporation, 241; Membership Committee, 99; New Members Admitted, 1, 98, 99, 145, 204, 434; Representatives: on Council of American Association for the Advancement of Science, 100; on International Mathematical Union, 100; on National Research Council, 146, 188, 205; San Francisco Section, Meetings, 10, 188, 193, 425; Officers, 10, 333; Southwestern Section, Meetings, 125, 378; Officers, 125; Statistics, 100; Summer Meeting, 99, 141, 146, 188, 435; Transactions, 41, 205, $235,283,378,425,481$.

Associations for the Advancement of Science: American, 91, 100; British, 41.

American Society of Civil Engineers, 235; Belgian Academy, 425; Berlin Academy, 141, 284; Bologna Academy, 425; Cracow Academy, 333; Deutsche Mathematiker-Vereinigung, 333; Friends of Philosophy "Als Ob," 481: International Mathematical Congress, 188, 435; International Mathematical Union, 100, 188; Italian Society of Sciences, 425; Jablonowski Society, 41; Mathematical Association of America, 141, 188, 433; National Academy of Sciences, 284; National Research Council, 41, 146, 188, 189, 205, 284; Paris Academy, 91; Royal Astronomical Society, 141; Société Mathématique de France, 425; Unione Matematica Italiana, 91; Vienna Academy, 481.

Books, Announcement of New, 141, 189, 283, 284, 426.

Doctorates in Mathematics, American, 235, 236.

Journals:

American Journal of Mathematics, 91, 141, 283, 378; Annali di Matematica, 41, 188; Annals of Mathematics, 283, 378, 425; Bell System Technical Journal, 91; Bibliotheca Mathematica, 481; Bollettino della Unione Matematica Italiana, 91; Jahrbuch über die Fortschritte der Mathematik, 284; Jahresbericht der Deutschen Mathematiker-Vereinigung, 333, 426, 481; Mathematische Annalen, 426; Mathematische Zeitschrift, 426; Scientia, 141; Transactions of the American Mathematical Society, 41, 205, 235, 283, 378, 425, 481.

Papers and Communications Presented to the Society, Authors:

Alexander, J. W., 116, 148; Allen, E. S., 106; Archibald, R. C., 435. Barnett, I. A., 203; Barter, J. D., 10; Bell, E. T., 11, 11, 107, 193, 194; Bernstein, B. A., 11, 12, 194, 436; Birkhoff, G. D., 101; Blichfeldt, H. F., 194, 217; Bliss, G. A., 101, 206; Blumberg, H., 203; Brahana, H. R., 198; Bray, H. E., 107, 210; Bridgman, P. W., 101; Brinkmann, H.'W., 106, 215; Brown, B. H., 103; Brown, E. P., 102; Burgess, R. W., 108.

Cajori, F., 12, 101, 107, 195; Camp, B. H., 109; Camp, C. C., 124; Candy, A. L., 125; Carpenter, A. F., 195; Chittenden, E. W., 123; Coble, A. B., 120; Coolidge, J. L., 106; Court, N. A., 442; Cowley, E. B., 9, 115; Craig, C. F., 107; Crum, W. L., 7, 8, 206.

Davis, H. T., 119, 199; Dickson, L. E., 120, 121, 200; Dines, L. L., 440; Dodd, E. L., 216; Douglas, J., 4, 5, 209; Dresden, A., 121.

Edington, W. E., 122; Eiesland, J., 443, 443; Eisenhart, L. P., 212, 213; Emch, A., 149; Evans, G. C., 210, 444. 
Feldstein, M. M., 199; Foster, M. C., 215; Franklin, P., 106, 214. Garabedian, C. A., 146, 211; Glenn, O. E., 110, 151, 208, 437; Graustein, W. C., 104, 212, 441; Gronwall, T. H., 208, 218.

Haskins, C. N., 208; Hazlett, O. C., 109; Hedrick, E. R., 127, 437; Hille, E., 6, 7, 208; Hitchcock, F. L., 115; Hollcroft, T. R., 147, 217; Hoskins, L. M., 13; Huntington, E. V., 207; Hutchinson, J. I., 208. Ingold, L., 127, 437; Ingraham, M. H., 199.

Jackson, D., 5, 121, 201.

Kakeya, S., 153; Kasner, E., 3, 150; Kazarinoff, D. C., 202, 440; Kellogg, O. D., 207; Kendall, C., 125; Koopman, B. O., 104.

Lane, E. P., 117; Lefschetz, S., 126, 197, 199; Lehmer, D. N., 195; Levy, H., 213; Linfield, B. Z., 5, 6, 107; Lipka, J., 104, 152, 216; Logsdon, M. I., 119.

McDonnell, J., 444; MacDuffee, C. C., 111, 209; Marriott, R. W., 109; Mathews, R. M., 8; Michal, A., 210, 444; Miller, G. A., 110, 111, 199; Moore, C. L. E., 104; Moore, C. N., 111, 439; Moore, R. L., 108, 119, 125, 438; Mordell, L. J., 435; Morse, H. C. M., 112; Moulton, F. R., 201; Murnaghan, F. D., 442; Murray, F. H., 116, 149, 441.

Neikirk, L. I., 195; Nelson, C. A., 150; Nörlund, N. E., 212.

Pfeiffer, G. A., 4, 151; Phillips, H. B., 112; Poritsky, H., 115; Post, E. L., 9, 113, 114.

Rainich, G. Y., 148, 213, 437, 441; Raynor, G. E., 116; Rice, L. H., 6, 153; Richardson, R. G. D., 439; Rietz, H. L., 197; Ritt, J. F., 147; Roever, W. H., 126; Roman, I., 201; Rutledge, G., 113,152 .

Safford, F. H., 212; Schwatt, I. J., 115; Shapley, H., 101; Silverman, L. L., 112; Sinclair, M. E., 438; Smail, L. L., 12, 196; Smith, E. R., 126; Smith, G. W., 125, 126; Sperry, P., 196.

Taylor, J. S., 105; Thomas, J. M., 443; Thomas, T. Y., 218; Turner, J. S., 127, 202.

Vandiver, H. S., 436; Veblen, O., 3, 101, 218.

Walsh, J. L., 102, 150, 211, 442; Weisner, L., 8, 113, 151; Whelan, A. M., 147; White, H. S., 436; Wiener, N., 105, 106, 211, 440; Wilder, R. L., 118, 152; Williams, A. R., 13; Williams, K. P., 124; Wood, F. E., 198, 202.

Zeldin, S. D., 217.

\section{Personal Notes:}

Abraham, M., 237; Adams, C. R., 42, 236; Albanese, G., 427; Albert, O. W., 482; Alderton, N. M., 428; Allen, B. R., 428; Anderegg, F., 42; Anning, N., 380; Antoine, L., 91; Archibald, R. C., 379; Armellini, G., 379; Armentrout, W. E., 428; Arnold, H. E., 380; Ashton, C. H., 283.

Bain, G. W., 380; Baker, H. F., 284; Ballantine, J. P., 380; Barnett, I. A., 380; Barnett, S. J., 188; Batchelder, P. M., 380; Bateman, H., 283; Bedarida, M., 427; Béghin, H., 91; Bell, E. T., 283; Bernstein, B. A., 333; Bill, E. G., 142; Birkhoff, G. D., 92, 379; Blakslee, T. M., 380; Blichfeldt, H. F., 283; Bliss, G. A., 100; Böhm, F., 481; Bohr, N., 426, 482; Bompiani, E., 379; Boulad, F., 91; Bragg, W. H., 142; Brandner, F. A., 189; Brannon, M. A., 142; Brenke, W. C., 283; Brocard, P., 42; Brouse, D., 189; Brown, B. H., 236; Brown, E. W., 92, 188, 284; Bruck, P., 42; Bryant, F. N., 237; Buchanan, D., 283; Buchanan, M., 236; Buchholz, H. F., 42; Burkill, J. C., 235; Butts, W. H., 42.

Cahen, 141; Cain, W., 235; Cajori, F., 235; Camichel, C., 92; Camp, B. H., 427; Campbell, A. D., 482; Campbell, W. B., 285; Capron, P., 283; Ćarey, F. S., 284; Carr, F. E., 237, 380; Carscallen, 
G. E., 482; Cartan, E., 141; Carus, E. H., 236; Cecioni, F., 427; Cederberg, W. E., 236; Chandler, H. W., 380; Chantry, A. J., 380; Chazy, J., 92; Cherubino, S., 427; Cipolla, M., 427; Cisotti, U., 426; Clarke, E. H., 236; Cohn, F., 237; Cole, F. N., 2, 14, 482; Cole, L. R., 142, 428; Collingwood, E. F., 235; Comessati, A., 379; Conwell, H. H., 142; Cragwell, J. A., 482; Crum, W. L., 333; Cullis, C., 427; Curtiss, D. R., 283; Cushing, E. P., 92.

Dadourian, H. M., 284; Daniell, P. J., 379; Darwin, C. G., 92; Davis, B. 188; Davis, H. T., 428; Davis, J. E.. 427; Dean, W. R., 235; Decherd, M. E., 380; Demaree, R. G., 189; Denjoy, A., 141; Denston, W., 42; DePorte, J. V., 380; Dodd, E. L., 380; Doole, H. P., 189; Dostal, B. F., 92, 428; Drach, J., 91; Dresden, A., 283; Duane, W., 188.

Echols, W. H., 283; Edwards, C. C., 189; Eiesland, J., 283; Einstein, A., 92, 235; Eisenhart, L. P., 205, 481; Eshleman, J. D., 236; Ettlinger, H. J., 380; Evans, G. C., 283; Everett, H. S., 236.

Fairchild, J. T., 285; Favaro, A. N., 42; Feldstein, M. M., 428; Field, F., 142; Fields, J. C., 283; Finkel, B. F., 379; Finsler, P., 237; Fiske, T. S., 92; Fite, W. B., 427; Foote, P. D., 188; Fort, T., 283; Francis, E. C., 235; Franklin, P., 285; Frost, E. B., 188; Fulmer, H. K., 142.

Garabedian, C. A., 380; Garabedian, H. L., 285; Garrison, D. M., 379; Giese, H., 189; Gokhale, V. D., 236, 427; Gorrell, G. W., 285; Gosse, 284; Gow, J., 237; Griffing, B., 142.

Halbert, K. W., 285; Hamilton, W. A., 142, 333; Hancock, H., 91, 283; Harkness, J., 283; Hartogs, F., 142; Haskell, M. W., 283; Haskins, C. N., 205; Hawkes, H. E., 283; Hazlett, O. C., 481; Hecken, H. P., 142; Hedrick, E. R., 283; Henderson, A., 427; Herglotz, G., 481; Heun, K., 237; Hilb, E., 481; Hill, M. A., 189; Hill, M. J. M., 284; Hille, E., 481, 482; Hitt, J. R., 142; Hobson, E. W., 284; Hoepli, U., 42; Holl, D. L., 482; Holman, M., 428; Howard, P. F., 237; Hughes, W. L., 285; Hull, D., 142; Huntington, E. V., 146, 188; Huntley, H., 237; Hutchinson, A., 237.

Ingham, A. E., 235.

Jackson, D., 283; James, C. G. F., 235; Janes, W. C., 189; Jarolimek, C., 285; Jeffery, R. L., 285; Jones, D. C., 285; Jones, S. I., 428; Julia, G., 427.

Kasner, E., 205, 481; Keller, S. S., 42; Kendall, C., 236; Kennon, R. E., 380; Kholodovsky, E. A., 427; Killam, S. D., 380; Killing, W., 380; Kimball, B. F., 285, 428; Kimball, W. S., 236; Kirchner, W. H., 427; Kneser, A., 481; Knott, C. G., 42; Koebe, C., 235; Konantz, E. L., 92; Krafft, M., 427; Krüger, L., 482.

Lane, E. P., 333; Langer, R. E., 236; Langevin, P., 235; Langman, H., 236; Laura, E., 379; Leathem, J. G., 285; Lehmer, D. N., 333; Lennes, N. J., 283; Lerch, M., 285; LeRoux, J., 91; Levett, R., 237; Levi-Civita, T., 426; Ievinson, H. C., 237; von Lindemann, F., 426; Linehan, P. H., 283; Little, C. N., 482; Longley, W. R., 283; Lorentz, H. A., 237, 426; Loria G., 92; Love, A. E. H., 426; Löwner, K., 481; Ludendorf, H., 235; Lyman, T., 188; Lyons, W. H., 42.

McCurry, C. E., 285; McGarry, L. J., 237; McSweeny, A. A., 428; MacDuffee, C. C., 236, 482; MacQueen, M. L., 428; Maizlish, I., 380; Mallison, 481; Markley, J. L., 283; Marshall, J., 427; Mason, M., 284; Meacham, E. D., 236; Metzler, W. H., 427; Michelson, 92, 141, 284, 284; Miles, F. R. C., 142, 285; Miller, G. A., 91, 283; Mills, C. N., 380; Milne, T. H., 380; Milne, W. E., 283; Montel, P., 141; Moore, C. N., 100; Moore, E. H., 91; Moore, R. L., 380; Mordell, L. J., 333; Morenus, E. M., 236; Morley, F., 283; Morley, R. K., 283; Mossman, T. A., 189; Muehlman, P., 285; Mullikin, A. M., 236; Mullins, G. W., 237; Myrberg, P. J., 41. 
Newman, M. H. A., 235; Niven, C., 333; Nordgaard, M. A., 142. Olds, G. D., 283, 379; O'Leary, E. J., 142; Osborn, J. O., 380.

Page, L., 188; Pairman, E., 236; Palatini, A., 379; Pascal, B., 284; Pascal, M., 427; Pehrson, E. W., 380; von Peinthner-Lichtenfels, O., 482; Perkins, F. W., 285; Perrin, J., 425; Pettit, H. P., 236; Pierce, J. E., 428; Pincherle, S., 91; Pitcher, A. D., 428; Poor, C. L., 92; Porter, M. B., 141; Porter, W. L., 285; Pound, V. E., 92; Prandtl, L., 41; Proudman, J., 235; Pupin, M. I., 284.

Randall, H. M., 188; Raveau, C., 92; Raynor, G. E., 482; Rechard, O. H., 284; Reed, F. W., 428; Reed, M., 189; Reid, C., 42; Reilly, J.F., 236; Richard, P., 92; Richardson, R. G. D., 100, 283; Richmond, H. W., 426; Richtmyer, F. K., 188; Rietz, H. L., 188, 283; Riley, J. L., 285; Robinson, P. O., 189; Robison, G. M., 482; Roman, I., 482; Rosanes, J., 42; Rosati, C., 379; Rosenbach, J. R., 284; Rowe, W. H., 189; Rutherford, E., 41, 284; Rutledge, G., 428.

Safford, F. H., 283; Sannia, G., 379; Sbrana, F., 427; Schlesinger, F., 91; Schur, A., 427; Scorza, G., 425; Segre, C., 41; Seiler, E. F., 285; Sherrington, C. S., 41; Sherwood, G. E. F., 283; Shields, W. F., 237; Sinclair, M. E., 42; Slaught, H. E., 283; Slichter, L., 237; Slobin, H. L., 237; Slotnik, M. M., 285; Smail, L. L., 237; Smith, C. E., 283; Smith, P. K., 285; Smith, T. L., 285; Snyder, V., 41; Sommerfeld, A., 142; Soreau, R., 92; Springer, J., 426; Steinmetz, C. P., 482; Stephens, R. P., 142; Steward, G. C., 427; Stone, R. B., 283; Stoneley, R., 427; Stout, C. E., 428; Stromgren, E., 481; Study, E., 481; Stuyvaert, M., 141; Swann, W. F. G., 188; Swartzel, K. D., 283.

Tanner, J. H., 283; Thompson, R., 285; Thomson, J., 284, 426; Tisdale, W. E., 188; Titsworth, A. A., 285; Togliati, E. G., 141; Tonelli, L., 425; Turner, B. M., 427; Turner, J. S., 236; Tyler, H. W., 283.

Udinski, W. P., 236.

Veblen, O., 41, 91, 92, 188, 188, 283; Venn, J., 285; Villat, H., 426; Vitali, G., 379.

van der Waals, J. D., 285; Weaver, W., 237; Weber, W. W., 428; Webster, A. G., 285; Webster, D. L., 284; Weisner, L., 284; Wells, V. H., 142; White, H. S., 283; Whittaker, E. T., 285; Wilbur, W. E., 237; Willard, J. M., 482; Wilson, D. T., 482; Wilson, W. H., 380; Wong, B. C., 236; Wood, F., 380; Wood, R. B., 189; Wylie, C. C., 42 .

Yeaton, C. H., 380; Yoder, P. Y., 380; Young, J. W., 283, 333.

Zeisler, E. B., 236.

Prizes and Medals:

Ackermann-Teubner, 235; American Society of Civil Engineers, Croes Medal, 235; Belgian Academy, 425; Berlin Academy: Steiner, 141; Bôcher, 188; Bologna Academy, 425; Cambridge University: Adams, Smith, Rayleigh, 235; Mayhew, 481; Cole, 2, 14, 205; Italian Society of Sciences, 425; Jablonowski Society, 41; Lasserre, 235; Paris Academy: Francœur, Grand, Montyon, de Parville, Poncelet, 91; Binoux, Houllivigue, Hughes, Montyon, Valz, 92; Royal Astronomical Society, 141; James Scott Medal, 426; Vienna Academy, 481.

Universities and Technical Schools:

Amherst, 482;

California, 142;

Cambridge, 235, 481;

Chicago, 189, 331;

Columbia, 331;

Cornell, 331;

Harvard, 332, 482;
Illinois, 332;

Massachusetts Institute, 332;

Pennsylvania, 332;

Princeton, 333;

Texas, 141 ;

Wisconsin, 379;

Yale, 379,482 . 